Serafim Barbosa Santos Filho 1,2 Sandhi Maria Barreto 3

\section{Atividade ocupacional e prevalência de dor osteomuscular em cirurgiões-dentistas de Belo Horizonte, Minas Gerais, Brasil: contribuição ao debate sobre os distúrbios osteomusculares relacionados ao trabalho}

\author{
Occupational activity and prevalence of upper-limb \\ and back pain among dentists in Belo Horizonte, \\ Minas Gerais State, Brazil: a contribution to the \\ debate on work-related musculoskeletal disorders
}

1 Departamento de Medicina Preventiva e Social, Faculdade de Medicina, Universidade Federal de Minas Gerais. Av. Alfredo Balena 190, Belo Horizonte, $M G$ 30110-100, Brasil. serafim@dedalus.lcc.ufmg.br

2 Secretaria de Saúde de Belo Horizonte. Av. Pedro II 307, Belo Horizonte, $M G$ 30710-010, Brasil. sbarreto@cpqrr.fiocruz.br

3 Laboratório de Epidemiologia e Antropologia Médica, Centro de Pesquisa René Rachou, Fundação Oswaldo Cruz. Av. Augusto de Lima 1715, Belo Horizonte, $M G$ 30190-002, Brasil.

\begin{abstract}
The objective was to determine the prevalence of upper-limb and back pain among dentists ( $n=358$ ) and factors associated with these symptoms. Dentists were interviewed using $a$ self-administered questionnaire containing data on sociodemographic, occupational, lifestyle, and psychosocial factors and presence, site, and characteristics of pain. Participation rate was 92.3\%. 58\% reported upper limb pain, with 22, 21, 20, and 17\% for the arm, back, neck, and shoulder, respectively. $26 \%$ reported daily frequency and $40 \%$ classified pain as moderate or severe. In the multivariate analysis (multiple logistic regression), the factors associated with pain were: neck: anxiety/depression $(O R=2.3 ;$ CI95\%: 1.2-4.5), compressor in the office $(O R=2.1 ; C I 95 \%$ : 1.2-3.7), job satisfaction (OR = 0.3; CI95\%: 0.1-0.9), and use of indirect vision (OR = 0.5; CI95\%: 0.3-0.9); shoulder: income $>20$ minimum wage (OR =2.9; CI95\%: 1.2-6.7), greater productivity $(O R=3.3$; CI95\%: 1.3-8.4), height $\geq 160 \mathrm{~cm}(\mathrm{OR}=0.3$; CI95\%: 0.2-0.7), and age 30-49 years $(\mathrm{OR}=0.3$; CI95\%: 0.1-0.8); back: anxiety/depression (OR = 2.3; CI95\%: 1.2-4.5), manual activity $(O R=0.4$; CI95\%: 0.2-0.9), and being married (OR =0.5; CI95\%: 0.3-0.9); arms: manual activity $(O R=1.8$; CI95\%: $1.0-3.2)$.
\end{abstract}

Key words Repetition Strain Injury; Work-related Musculoskeletal Disorders; Occupational Health

Resumo Estudo de prevalência de dor osteomuscular e fatores associados ao sintoma em cirurgiões-dentistas ( $n=358)$, utilizando-se de questionário auto-aplicável, com dados sócio-demográficos, ocupacionais, psicossociais, hábitos de vida, localização e características de dor. 92\% dos dentistas selecionados participaram do estudo. A prevalência de dor no segmento superior foi de 58\%: 22\% de dor no braço, $21 \%$ na coluna, $20 \%$ no pescoço e $17 \%$ no ombro; $26 \%$ relataram dor diária e 40\% dor moderada/forte. Na análise multivariada (regressão logística múltipla), os fatores associados à dor foram: Pescoço: ansiedade/depressão (RC=2,3; IC95\%: 1,2-4,5), ruídocompressor ( $R C=2,1$; IC95\%: 1,2-3,7), satisfação no trabalho $(R C=0,3$; IC95\%: 0,1-0,9) e uso de visão indireta ( $R C=0,5$; IC95\%: 0,3-0,9); ombro: renda $>20$ salários $(R C=2,9 ; I C 95 \%: 1,2-6,7)$, maior produtividade ( $R C=3,3$; IC95\%: 1,3-8,4), altura $\geq 160 \mathrm{~cm}(R C=0,3 ;$ IC95\%: 0,2-0,7) e idade 30-49 anos ( $R C=0,3$; IC95\%: 0,1-0,8); coluna: ansiedade/depressão ( $R C=2,3$; IC95\%: 1,2-4,5), atividade manual ( $R C=0,4$; IC95\%: 0,2-0,9) e ser casado ( $R C=0,5$; IC95\%: 0,3-0,9); braços: atividade manual ( $R C=1,8 ;$ IC95\%: 1,0-3,2).

Palavras-chave Traumatismo por Distensão Repetida; Distúrbios Osteomusculares Relacionados ao Trabalho; Saúde Ocupacional 


\section{Introdução}

A odontologia tem sido considerada uma profissão "estressante", sendo freqüentemente associada a agravos à saúde, tanto de ordem física como psíquica (Nogueira, 1983; Coster et al., 1987; Mandel, 1993; Medeiros \& Riul, 1994; Lopes \& Neto, 1994). Estudos sistemáticos sobre os distúrbios músculo-esqueléticos em cirurgiões-dentistas vêm sendo realizados desde a década de 50 (Rundcrantz et al., 1991a), e são responsáveis pelas primeiras propostas de modificações no processo de trabalho dos dentistas, inclusive a mudança do trabalho da posição ortostática para a posição sentada. Entretanto, o conhecimento sobre esses problemas ainda é muito incipiente, deixando muitas lacunas na compreensão da história natural dos quadros clínicos.

A maioria dos estudos epidemiológicos existentes sobre o assunto são estudos seccionais, variando muito tanto no método de identificação e classificação dos diversos sintomas osteomusculares como no tratamento dos dados. Os trabalhos mais recentes descrevem sintomas de dor e desconforto em diferentes regiões do segmento superior do corpo (Bauer, 1985; Ekenvall et al., 1990; Rundcrantz et al., 1991b; Travassos, 1995; Cherniack, 1996; Santos Filho \& Barreto, 1998b) e colocam os profissionais de odontologia entre os primeiros lugares em afastamentos do trabalho por incapacidade temporária ou permanente (Doorn, 1995), respondendo por cerca de $30 \%$ das causas de abandono prematuro da profissão (Ferreira, 1997b). A maioria dos quadros descritos pode ser enquadrada entre os Distúrbios Osteomusculares Relacionados ao Trabalho (DORT), denominação mais atual para as chamadas Lesões por Esforços Repetitivos (INSS, 1998).

As regiões mais acometidas são o pescoço, ombro e coluna lombar, sendo grande a variação nas taxas de acometimento entre os cirurgiões-dentistas. Considerando isoladamente essas regiões, as taxas de prevalência variam de 36 a 57\% na região lombar (Letho et al., 1991; Doorn, 1995); 42\% no ombro (Letho et al., 1991) e $44 \%$ na região cervical (Milerad \& Ekenvall, 1990; Rundcrantz et al., 1991b). O acometimento de mais de um local, simultaneamente, oscila bastante, sendo descritas taxas que variam de 51 a $90 \%$ para o pescoço e ombro (Milerad \& Ekenvall, 1990; Rundcrantz et al., 1991a, 1991 b) e de 30 a $72 \%$ para esses dois segmentos mais a região lombar (Rundcrantz et al., 1991a; Mandel, 1993). Alguns trabalhos encontraram maior freqüência de sintomas entre os dentistas quando comparados com a popula- ção geral (Kelsey, 1982; Letho et al., 1990) ou com outros profissionais de saúde (Akesson et al., 1995; Doorn, 1995). A chance de apresentar sintomas osteomusculares foi 12 vezes maior em cirurgiões-dentistas quando comparados aos profissionais de farmácia, em um estudo na Suécia (Milerad \& Ekenvall, 1990).

Os fatores que têm sido associados aos quadros músculo-esqueléticos abrangem componentes do processo de trabalho, englobando a atividade repetitiva, monótona e o esforço físico-postural, aspectos da organização do trabalho e fatores psicossociais. Insuficiência de pausas, insatisfação no trabalho e remuneração por assalariamento foram observados entre os ciurgiões-dentistas que apresentaram mais queixas (Letho et al., 1990; Rundcrantz et al., 1990, 1991a, 1991b; Doorn, 1995). O uso de recursos para melhorar a execução de procedimentos, como o espelho para cavidade oral e posições de trabalho mais confortáveis, foi mais comum entre os dentistas que manifestaram menos sintomas (Rundcrantz et al., 1990). Parece também haver correlação entre indicadores de estresse e sintomas músculo-esqueléticos, evidenciados por associações entre esses distúrbios e maiores escores de estresse (Letho et al., 1990; Murtomaa et al., 1990).

Apesar das altas taxas de prevalência de queixas osteomusculares descritas em outros países, muito pouco se conhece sobre esses sintomas entre os cirurgiões-dentistas no Brasil. No presente estudo, investigaram-se a prevalência e as características desses sintomas entre dentistas da rede pública de Belo Horizonte, Minas Gerais e os fatores a eles associados. Esta pesquisa está inserida no debate mais amplo, embora ainda incipiente e genérico, sobre os DORT.

\section{Material e métodos}

O universo de estudo foi composto pela totalidade dos dentistas vinculados ao Serviço Público de Saúde de Belo Horizonte (SUS/BH), trabalhando na clínica odontológica, inclusive aqueles afastados temporariamente por gozo de férias ou outro motivo. Os dentistas vinculados ao SUS/BH distribuem-se em cerca de 225 locais de trabalho, incluindo escolas públicas e centros de saúde.

A coleta de dados foi realizada por meio de questionário auto-aplicável, contendo informações sócio-demográficas, aspectos ergonômicos e do ambiente de trabalho (equipamentos, métodos e instrumentos utilizados, condições físicas do local), organização do trabalho 
(jornada, sistema utilizado, ritmo, produção), hábitos pessoais e fatores individuais (fumo, álcool, esporte, lazer, atividades extra-ocupacionais, sono, idade, estatura, peso), características psicológicas e psicossociais (perfil de personalidade, nível de satisfação no trabalho, remuneração e lazer) e histórico de saúde (queixa atual, caracterização do sintoma, procura de assistência, diagnóstico, tratamentos, limitações impostas e afastamentos do trabalho). As perguntas relativas ao ambiente e processo de trabalho previam uma resposta para cada local, quando o dentista trabalhava em mais de um serviço. As questões referentes à localização da dor foram abertas, mas tinham como referência uma lista com todas as regiões anatômicas detalhadas e uma numeração correspondente para facilitar e padronizar as respostas.

Junto com os questionários foi enviada uma correspondência explicando o estudo e o compromisso com a confidencialidade dos dados e solicitando a colaboração dos dentistas. A maioria dos questionários foi distribuída e recolhida mediante malotes oficiais dos serviços da Secretaria de Saúde, e os demais diretamente ou por correio. A pesquisa de campo ocorreu entre maio e setembro de 1997, sendo organizado banco de dados com o uso do programa Epi-Info 6.04 (CDC/WHO, 1996).

As informações relacionadas à satisfação com o trabalho foram obtidas usando-se uma escala de nota de 0 a 10, variando do menor para o maior nível de satisfação. Alguns dados como idade, peso, altura e tempo de trabalho foram colhidos com a maior precisão possível e reagrupados em categorias para evitar uma dispersão muito grande das informações e uma instabilidade dos coeficientes estimados.

\section{Análise dos dados}

Foi solicitado aos participantes com dor que indicassem a região em que era mais acentuada. Esses locais foram agrupados pelas seguintes regiões do segmento superior: pescoço, ombro, coluna (torácica e/ou lombar) e membro superior (englobando dedos, mãos, punhos, cotovelo, antebraço e/ou braço).

A prevalência de dor músculo-esquelética atual foi determinada para cada região. $\mathrm{O}$ mesmo indivíduo poderia oferecer uma resposta afirmativa para dor em uma ou mais regiões, caso esse sintoma abrangesse mais de um local. Os fatores associados à dor músculo-esquelética em cada uma das quatro regiões definidas foram investigados por intermédio de regressão logística múltipla não condicional, comparando-se a chance de exposição entre casos e não-casos. Foram definidos como casos os indivíduos que relataram dor atual em cada região, independente de sua intensidade e duração. Os demais indivíduos sem relato de dor naquela região foram considerados não-casos.

No modelo multivariado, foram incluídas todas as variáveis que apresentaram associação ao nível de $\mathrm{p}<0,10$, além do sexo e faixa etária, independentemente de estarem estatisticamente associados à queixa de dor, por serem potenciais fatores de confusão na relação entre a variável independente e a dependente (Clayton \& Hills, 1996). Retiveram-se no modelo final apenas as variáveis que permaneceram associadas de forma independente, $\mathrm{p}<0,05$, à queixa de dor osteomuscular, após o ajuste pelo efeito das outras variáveis no modelo. As análises uni e multivariadas foram feitas com a utilização do pacote estatístico Stata (Stata Corporation, 1997).

\section{Resultados}

Do total de 388 questionários enviados, foram respondidos 361, sendo 358 utilizados na análise. Três questionários foram eliminados por estarem incompletos e faltarem informações essenciais para identificação dos mesmos, resultando em um índice de participação de 92,3\%. Não houve diferença estatisticamente significante entre os dentistas que participaram e os que não participaram do estudo quanto ao local e regime de trabalho.

\section{Principais características}

da população do estudo

A maioria da população do estudo era do sexo feminino, representando $66 \%$ dos participantes; $33 \%$ eram do sexo masculino. A idade média dos dentistas foi de 41 anos (DP $=8,5$ ), estando a maior parte nas faixas etárias consideradas produtivas, até 50 anos (85\%). Sessenta e cinco por cento eram casados e a maioria (62\%) tinha filhos. Na distribuição por faixa salarial, $35 \%$ ganhavam entre 5 e 10 salários mínimos, $38 \%$ de 11 a 20 , e $21 \%$ tinham renda superior a 20 salários mensais.

$O$ índice médio de massa corporal encontrado foi de $23,5 \mathrm{~kg} / \mathrm{m}^{2}(\mathrm{DP}=4)$, estando a maioria (67\%) dentro dos padrões considerados normais, entre 19 e $25 \mathrm{~kg} / \mathrm{m}^{2}$. Oitenta e quatro por cento dos dentistas não fumavam e metade não consumia bebida alcoólica.

A média de tempo de trabalho na profissão foi de 16 anos ( $\mathrm{DP}=7$ ), variando de 2 a 44 anos, e mais da metade (54\%) tinha acima de 15 anos 
Tabela 1

Distribuição dos dentistas do estudo segundo variáveis sócio-demográficas, local e tempo de trabalho e hábitos pessoais. Belo Horizonte, Minas Gerais, 1997.

\begin{tabular}{|c|c|c|}
\hline Variável & $\begin{array}{l}\text { Freqüência } \\
\text { (total }=358 \text { ) }\end{array}$ & Percentual (\%) \\
\hline \multicolumn{3}{|l|}{ Sexo } \\
\hline Masculino & 117 & 32,7 \\
\hline Feminino & 238 & 66,5 \\
\hline Não informado & 3 & 0,8 \\
\hline \multicolumn{3}{|l|}{ Faixa etária } \\
\hline Até 29 anos & 31 & 8,6 \\
\hline 30 a 39 anos & 141 & 39,4 \\
\hline 40 a 49 anos & 132 & 36,9 \\
\hline 50 anos e mais & 51 & 14,2 \\
\hline Não informado & 3 & 0,8 \\
\hline \multicolumn{3}{|l|}{ Estado civil } \\
\hline Casado & 231 & 64,5 \\
\hline Solteiro & 94 & 26,3 \\
\hline Outros & 33 & 9,2 \\
\hline \multicolumn{3}{|l|}{$\begin{array}{l}\text { Índice de massa corporal } \\
\text { (Peso/Altura } 2 \text { ) }\end{array}$} \\
\hline $\begin{array}{l}\text { Até } 19 \\
20 \text { a } 24\end{array}$ & $\begin{array}{r}45 \\
213\end{array}$ & $\begin{array}{l}12,6 \\
59,5\end{array}$ \\
\hline 25 a 29 & 74 & 20,7 \\
\hline 30 e mais & 26 & 7,3 \\
\hline Não informado & 6 & 1,7 \\
\hline \multicolumn{3}{|l|}{ Fumo } \\
\hline Não & 300 & 83,8 \\
\hline Sim & 50 & 14,0 \\
\hline Não informado & 8 & 2,2 \\
\hline \multicolumn{3}{|l|}{ Bebida alcoólica } \\
\hline Não & 178 & 49,7 \\
\hline Sim & 170 & 47,5 \\
\hline Não informado & 10 & 2,8 \\
\hline \multicolumn{3}{|l|}{ Local de trabalho } \\
\hline Apenas Público (SUS/BH) & 86 & 24,0 \\
\hline SUS/BH e outros & 272 & 76,0 \\
\hline \multicolumn{3}{|l|}{$\begin{array}{l}\text { Tempo de trabalho como } \\
\text { dentista }\end{array}$} \\
\hline$<5$ anos & 21 & 5,9 \\
\hline 5 a 9 anos & 52 & 14,5 \\
\hline 10 a 14 anos & 84 & 23,5 \\
\hline 15 anos e mais & 194 & 54,2 \\
\hline Não informado & 7 & 2,0 \\
\hline \multicolumn{3}{|l|}{ Renda em salários mínimos } \\
\hline 5 a 10 & 126 & 35,2 \\
\hline 11 a 20 & 137 & 38,3 \\
\hline Mais de 20 & 74 & 20,7 \\
\hline Não informado & 21 & 5,9 \\
\hline
\end{tabular}

de exercício profissional. A maioria (76\%) dos dentistas atuava no setor público (SUS/BH) e outros locais de trabalho. A maior parte atendia população adulta e infantil (85\%). A caracterização da população estudada encontra-se na Tabela 1.

Prevalência e caracterização de dor músculo-esquelética no segmento superior

Cinqüenta e oito por cento dos dentistas apresentaram queixa de dor músculo-esquelética em uma ou mais regiões do segmento superior do corpo, dos quais $41 \%$ têm queixa em apenas uma região, $14 \%$ em duas e $3 \%$ em três locais. A dor no membro superior foi o principal sintoma relatado, com uma prevalência de $22 \%$. Em segundo lugar apareceu a queixa de dor na coluna torácica e/ou lombar, com prevalência de $21 \%$, prevalecendo a dor lombar. Em terceiro lugar apareceu o pescoço, com $20 \%$, seguido do ombro, com $17 \%$ de casos. (Tabela 2 ).

Foi feita uma avaliação da comorbidade entre as queixas por região, sendo encontradas as seguintes associações significativas ( $\mathrm{p}<$ 0,05): coluna-pescoço, na qual se observou que $29 \%$ dos indivíduos que tiveram dor na coluna também apresentaram queixa no pescoço; pescoço-ombro, mostrando que $25 \%$ dos casos de cervicalgia também apresentaram queixa no ombro; e coluna-membro superior, com percentual de $9 \%$ dos dentistas com dor na coluna apresentando dor concomitante no membro superior.

A maioria dos participantes qualificou a dor no segmento superior como leve ou moderada (42 e $45 \%$, respectivamente), e $12 \%$ qualificaram como forte. Quanto à freqüência, ressaltase a dor sentida diariamente $(26 \%)$ ou ao menos uma vez na semana $(27 \%)$.

A Tabela 2 apresenta as taxas de prevalência de dor e algumas características selecionadas. Cruzando as variáveis de intensidade e freqüência de dor, observam-se $40 \%$ de casos enquadrados como dor moderada ou forte com freqüência igual ou superior a uma vez na semana. Estes casos representaram em torno de $51 \%$ das queixas de dor no pescoço.

Quase a metade dos dentistas relata que a dor costuma levar à interrupção da sua atividade no trabalho, com alguma freqüência $(45 \%$, às vezes, e $3 \%$ sempre). Além disso, em $57 \%$ dos casos a dor levou a modificações nas rotinas de trabalho, lazer ou limitações da vida diária, como realização de cuidados pessoais e dirigir automóveis.

Vinte e três por cento dos dentistas com dor tinham história de afastamento do trabalho por 
Prevalência de dor osteomuscular (todas as localizações) e por região do segmento superior segundo características selecionadas e histórico de assistência, tratamento e afastamentos associados à dor. Belo Horizonte, Minas Gerais, 1997.

\begin{tabular}{|c|c|c|c|c|c|}
\hline \multirow[t]{2}{*}{ Característica da dor } & \multicolumn{5}{|c|}{ Prevalência de dor $(\%)(n=358)$} \\
\hline & $\begin{array}{l}\text { Todas as } \\
\text { localizações }\end{array}$ & $\begin{array}{l}\text { Membro } \\
\text { superior }\end{array}$ & Coluna & Pescoço & Ombro \\
\hline Queixa geral de dor & 58 & 22 & 21 & 20 & 17 \\
\hline Dor crônica (> 6 meses) & 77 & 82 & 80 & 77 & 69 \\
\hline $\begin{array}{l}\text { Dor moderada ou forte } \\
\text { ( } 1 \text { ou mais vezes por semana) }\end{array}$ & 40 & 36 & 35 & 51 & 44 \\
\hline Dor diária & 26 & 28 & 23 & 30 & 28 \\
\hline $\begin{array}{l}\text { Dor com limitação do trabalho, } \\
\text { lazer e/ou rotina diária }\end{array}$ & 57 & 59 & 56 & 54 & 64 \\
\hline \multirow[t]{2}{*}{ Histórico } & \multicolumn{5}{|c|}{ Percentual sobre total com dor $(n=209)$} \\
\hline & $\begin{array}{l}\text { Todas as } \\
\text { localizações }\end{array}$ & $\begin{array}{l}\text { Membro } \\
\text { superior }\end{array}$ & Coluna & Pescoço & Ombro \\
\hline Procurou assistência médica & 55 & 62 & 51 & 54 & 62 \\
\hline $\begin{array}{l}\text { Fez uso de medicamento } \\
\text { no último mês }\end{array}$ & 23 & 23 & 24 & 31 & 28 \\
\hline $\begin{array}{l}\text { Fez outro tipo de tratamento } \\
\text { (além do medicamentoso) }\end{array}$ & 31 & 35 & 25 & 32 & 33 \\
\hline Afastamento do trabalho & 24 & 36 & 13 & 23 & 25 \\
\hline
\end{tabular}

causa da dor, dentre estes, 39\% por mais de 15 dias. Mais da metade dos indivíduos sintomáticos (55\%) haviam procurado assistência médica e $23 \%$ haviam utilizado medicamento por causa da dor no último mês. Trinta e um por cento informaram outro tipo de tratamento.

Pôde-se observar que os indivíduos com sintoma no ombro apresentaram maiores limitações no trabalho e vida diária (64\%), acima do verificado em outras regiões; nos casos de dor no pescoço, $30 \%$ relataram sentir dor diariamente e $51 \%$ classificaram a dor como moderada ou forte, freqüente uma ou mais vezes por semana. Já as prevalências de dor diária (35\%) e moderada ou forte (25\%) na coluna foram menores que nas demais regiões, tendo levado a uma menor procura de assistência médica (51\%), menor utilização de medicamento (24\%) e também a um índice menor de afastamento do trabalho (13\%), sendo a maioria $(70 \%)$ desses afastamentos inferior a 15 dias.

Em relação à duração da dor, a grande maioria pode ser caracterizada como de evolução crônica, com $77 \%$ dos casos durando acima de seis meses (percentual que chega a $82 \%$ para a dor no membro superior). A duração da dor variou de 1 a 360 meses (mediana $=24$ ); entretanto, em $37 \%$ dos casos a duração foi inferior a um ano.
Nos quadros de dor com duração igual ou inferior a seis meses, concentraram-se maiores percentuais de dor leve (53\%) e de freqüência diária (35\%), do que nos casos mais crônicos, ou seja, com duração superior a seis meses (39 e 24\%, respectivamente). Quarenta por cento dos quadros agudos e crônicos relataram dor moderada ou forte que ocorria uma ou mais vezes por semana.

Além de dor, a maioria dos dentistas (54\%) apresentou também outro sintoma concomitante, do tipo dormência, sudorese, redução de força muscular, alteração de temperatura ou edema. Trinta e sete por cento relataram que foi realizado diagnóstico para sua queixa, aparecendo como descrições mais freqüentes os relatos de tensões musculares e algias decorrentes de posturas e esforço; processos inflamatórios do tipo tendinites e bursites; processos reumáticos, como artrites; e lesão por esforço repetitivo.

\section{Fatores associados à dor em cada região}

\section{- Membro superior}

Nas análises univariada e multivariada, o único fator que se mostrou significativamente associado à dor de membro superior $(p<0,05)$ foi 
a prática atual de atividade manual como tocar instrumentos musicais e tecer $(\mathrm{RC}=1,81$; IC95\%: 1,01-3,22) (Tabelas 3 e 4).

\section{- Coluna}

Os seguintes fatores foram identificados como significativamente associados à dor na coluna na análise univariada: tipo de equipo utilizado, com maior prevalência de queixa entre dentistas que trabalham com equipamentos mais antigos comparados aos que usam apenas os modelos mais novos ( $\mathrm{RC}=0,45$; IC95\%: 0,20-0,99); menor freqüência de dor em indivíduos que têm filhos (RC = 0,49; IC95\%: 0,29-0,82) ou que praticam atividade manual $(\mathrm{RC}=0,45$; IC95\%: 0,21-0,96); maior percentual de queixas entre os dentistas que relataram tendência à depressão, tensão, ansiedade e/ou preocupação excessiva (RC = 2,15; IC95\%: 1,16-3,98) (Tabela 3).

Os fatores que permaneceram associados à menor freqüência de dor no modelo final multivariado foram: prática de atividade manual atual ( $\mathrm{RC}=0,42$; IC95\%: 0,19-0,91); dentistas casados quando comparados aos solteiros ( $\mathrm{RC}=$ 0,52; IC95\%: 0,29-0,94). Já as pessoas mais depressivas, tensas ou muito preocupadas apresentaram maior freqüência de dor $(\mathrm{RC}=2,31$; IC95\%: 1,19-4,47) (Tabela 4).

\section{- Pescoço}

$\mathrm{Na}$ análise univariada, comparando com os dentistas que trabalham apenas nas posições consideradas mais adequadas para o tratamento do paciente (9 e 12 horas, ou seja, atrás e ao lado do paciente), os dentistas que relataram adoção de outras posições apresentaram maior queixa de dor no pescoço $(\mathrm{RC}=1,72$; IC95\%: 1,00-2,94). A presença de compressor dentro do consultório, que é o motor-fonte de energia para o funcionamento dos aparelhos, também foi associada a uma queixa de dor duas vezes maior quando comparado aos dentistas com compressor fora do consultório $(\mathrm{RC}=2,05$; IC95\%: 1,17-3,58). Os dentistas que usam ambas as visões, direta (olho nu) e indireta (com espelho), queixaram-se menos de dor no pescoço do que aqueles que utilizam apenas a visão direta ( $\mathrm{RC}=0,55$; IC95\%: 0,31-0,98).

As notas atribuídas ao grau de satisfação no trabalho foram inicialmente agrupadas em quatro níveis, mas devido ao tamanho da população estudada e às pequenas diferenças entre aqueles que deram notas maiores que 5, foram feitos reagrupamentos em apenas dois estratos (0-5 e 6-10). Maior satisfação no trabalho, considerada como os indivíduos que de- ram nota maior ou igual a seis, foi inversamente relacionada à queixa de dor no pescoço $(\mathrm{RC}=$ 0,35; IC95\%: 0,12-1,02).

Também aparece como fator significativamente associado a uma maior prevalência de dor no pescoço as referidas características psicológicas de depressão, tensão, ansiedade e/ou preocupação excessiva ( $\mathrm{RC}=2,42$; IC95\%: 1,27$4,63)$. Os resultados da análise univariada estão apresentados na Tabela 3.

Permaneceram associados à dor no pescoço no modelo final: local de instalação do compressor (RC = 2,09; IC95\%: 1,18-3,70); tipo de visão utilizada (RC = 0,51; IC95\%: 0,28-0,93); facilidade de depressão, tensão, ansiedade e/ou preocupação excessiva ( $\mathrm{RC}=2,30$; IC95\%: 1,18 4,47 ) e satisfação no trabalho, com os dentistas mais satisfeitos (nota maior ou igual a seis) apresentando menos queixas ( $\mathrm{RC}=0,28$; IC95\%: 0,09-0,87) (Tabela 4).

\section{- Ombro}

A prevalência de dor no ombro esteve associada na análise univariada com a faixa etária e a estatura. O sintoma foi menos freqüente entre os indivíduos de 30 a 49 anos, em relação aos mais jovens ( $\mathrm{RC}=0,41$; IC95\%: 0,17-0,95), não sendo significante para os acima de 50 anos, e também menos freqüente entre os dentistas mais altos, acima de 1,66 metro $(\mathrm{RC}=0,51$; IC95\%: 0,26-0,97) (Tabela 3). No modelo final multivariado, os fatores associados, de forma significante, com menor freqüência de dor no ombro foram: estatura superior a 1,66 metro $(\mathrm{RC}=0,32$; IC95\%: 0,15-0,68) e idade maior do que 50 anos (RC =0,31; IC95\%: 0,11-0,82). Já os fatores associados à maior freqüência de dor foram: atendimento de mais de sete pacientes por jornada de quatro horas de trabalho, comparando-se aos que atendem até quatro pacientes (RC = 3,28; IC95\%: 1,29-8,35); renda acima de 20 salários, comparados aos que recebem 10 salários mínimos ou menos $(\mathrm{RC}=2,87$; IC95\%: 1,23-6,67) (Tabela 4).

\section{Discussão}

As prevalências encontradas nesta investigação são próximas aos achados da literatura (Milerad \& Ekenval, 1990; Rundcrantz et al., 1990, 1991a, 1991b; Letho et al., 1991; Mandel, 1993; Doorn, 1995; Bammer, 1996), acrescentando-se o sintoma de membro superior, que se destacou em nosso estudo com taxa semelhante à das outras regiões. Os fatores associados à queixa de dor também foram consisten- 
Tabela 3

Fatores associados à dor músculo-esquelética, por região, entre os dentistas do estudo, na análise univariada.

Belo Horizonte, Minas Gerais, 1997.

\begin{tabular}{|c|c|c|c|c|}
\hline Região e fatores & Sem dor & Com dor & $\begin{array}{l}\text { Razão de } \\
\text { chance (RC) }\end{array}$ & IC (95\%) \\
\hline \multicolumn{5}{|l|}{ Membro Superior } \\
\hline \multicolumn{5}{|l|}{ Atividade manual atual } \\
\hline Não & 212 & 54 & 1,0 & \\
\hline Sim & 50 & 23 & 1,81 & $1,01-3,22$ \\
\hline \multicolumn{5}{|l|}{ Coluna } \\
\hline \multicolumn{5}{|l|}{ Tipo de equipo } \\
\hline Robot + Fixo + Kart & 203 & 61 & 1,0 & \\
\hline Robot + Fixo & 59 & 8 & 0,45 & $0,20-0,99$ \\
\hline \multicolumn{5}{|l|}{ Filhos } \\
\hline Não & 85 & 36 & 1,0 & \\
\hline Sim & 184 & 38 & 0,49 & $0,29-0,82$ \\
\hline \multicolumn{5}{|l|}{ Atividade manual atual } \\
\hline Não & 203 & 63 & 1,0 & \\
\hline Sim & 64 & 9 & 0,45 & $0,21-0,96$ \\
\hline \multicolumn{5}{|c|}{$\begin{array}{l}\text { Facilidade de depressão e/ou ansiedade } \\
\text { e/ou preocupação excessiva }\end{array}$} \\
\hline Não & 99 & 15 & 1,0 & \\
\hline $\operatorname{Sim}$ & 184 & 60 & 2,15 & $1,16-3,98$ \\
\hline \multicolumn{5}{|l|}{ Pescoço } \\
\hline \multicolumn{5}{|c|}{ Posições assumidas no trabalho (relógio) } \\
\hline 12 e 9 horas & 151 & 29 & 1,0 & \\
\hline Outras * & 120 & 41 & 1,72 & $1,00-2,94$ \\
\hline \multicolumn{5}{|c|}{ Satisfação no trabalho (nota) } \\
\hline Até 5 (não satisfeito) & 9 & 6 & 1,0 & \\
\hline 6 a 10 (satisfeito) & 278 & 65 & 0,35 & $0,12-1,02$ \\
\hline \multicolumn{5}{|l|}{ Instalação do compressor } \\
\hline Fora do consultório & 135 & 22 & 1,0 & \\
\hline Dentro do consultório & 141 & 47 & 2,05 & $1,17-3,58$ \\
\hline \multicolumn{5}{|l|}{ Tipo de visão utilizada } \\
\hline Direta & 60 & 23 & 1,0 & \\
\hline Direta + indireta & 217 & 46 & 0,55 & $0,31-0,98$ \\
\hline \multicolumn{5}{|c|}{$\begin{array}{l}\text { Facilidade de depressão e/ou ansiedade } \\
\text { e/ou preocupação excessiva }\end{array}$} \\
\hline Não & 101 & 13 & 1,0 & \\
\hline $\operatorname{Sim}$ & 186 & 58 & 2,42 & $1,27-4,63$ \\
\hline \multicolumn{5}{|l|}{ Ombro } \\
\hline \multicolumn{5}{|l|}{ Faixa etária } \\
\hline Até 29 anos & 22 & 9 & 1,0 & \\
\hline 30 a 49 anos & 234 & 39 & 0,41 & $0,17-0,95$ \\
\hline 50 anos e mais & 41 & 10 & 0,60 & $0,21-1,68$ \\
\hline \multicolumn{5}{|l|}{ Altura (em metros) } \\
\hline Até 1,60 & 92 & 25 & 1,0 & \\
\hline 1,61 a 1,65 & 63 & 15 & 0,90 & $0,43-1,79$ \\
\hline 1,66 e mais & 138 & 19 & 0,51 & $0,26-0,97$ \\
\hline
\end{tabular}

* Outras posições, além de 12 e 9 horas: (12 e 7), (9 e 7) ou (12, 9 e 7). 
Tabela 4

Fatores que permaneceram associados $(p<0,05)$ à dor músculo-esquelética entre dentistas na análise multivariada, por região. Belo Horizonte, Minas Gerais, 1997.

\begin{tabular}{|c|c|c|c|c|}
\hline Região e fatores & Sem dor & Com dor & $\begin{array}{l}\text { Razão de } \\
\text { chance (RC) }\end{array}$ & IC (95\%) \\
\hline \multicolumn{5}{|l|}{ Membro superior } \\
\hline \multicolumn{5}{|l|}{ Atividade manual atual } \\
\hline Não & 212 & 54 & 1,0 & \\
\hline Sim & 50 & 23 & 1,81 & $(1,01-3,22)$ \\
\hline \multicolumn{5}{|l|}{ Coluna } \\
\hline \multicolumn{5}{|l|}{ Atividade manual atual } \\
\hline Não & 203 & 63 & 1,0 & \\
\hline Sim & 64 & 9 & 0,42 & $0,20-0,91$ \\
\hline \multicolumn{5}{|c|}{$\begin{array}{l}\text { Facilidade de depressão e/ou ansiedade } \\
\text { e/ou preocupação excessiva }\end{array}$} \\
\hline Não & 99 & 15 & 1,0 & \\
\hline Sim & 184 & 60 & 2,31 & $1,20-4,47$ \\
\hline \multicolumn{5}{|l|}{ Estado civil } \\
\hline Solteiro & 69 & 25 & 1,0 & \\
\hline Casado & 191 & 40 & 0,52 & $0,29-0,94$ \\
\hline Outros & 23 & 10 & 0,9 & $0,36-2,46$ \\
\hline \multicolumn{5}{|l|}{ Pescoço } \\
\hline \multicolumn{5}{|c|}{ Satisfação no trabalho (nota) } \\
\hline Até 5 (não satisfeito) & 9 & 6 & 1,0 & \\
\hline 6-10 (satisfeito) & 278 & 65 & 0,28 & $0,09-0,87$ \\
\hline \multicolumn{5}{|l|}{ Instalação do compressor } \\
\hline Fora do consultório & 135 & 22 & 1,0 & \\
\hline Dentro do consultório & 141 & 47 & 2,09 & $1,18-3,70$ \\
\hline \multicolumn{5}{|l|}{ Tipo de visão utilizada } \\
\hline Direta & 60 & 23 & 1,0 & \\
\hline Direta + indireta & 217 & 46 & 0,51 & $0,28-0,93$ \\
\hline \multicolumn{5}{|c|}{$\begin{array}{l}\text { Facilidade de depressão e/ou ansiedade } \\
\text { e/ou preocupação excessiva }\end{array}$} \\
\hline Não & 101 & 13 & 1,0 & \\
\hline $\operatorname{Sim}$ & 186 & 58 & 2,30 & $1,18-4,47$ \\
\hline \multicolumn{5}{|l|}{ Ombro } \\
\hline \multicolumn{5}{|l|}{ Faixa etária } \\
\hline Até 29 anos & 22 & 9 & 1,0 & \\
\hline 30 a 49 anos & 234 & 39 & 0,31 & $0,11-0,82$ \\
\hline 50 anos e mais & 41 & 10 & 0,55 & $0,19-2,02$ \\
\hline \multicolumn{5}{|l|}{ Altura (em metros) } \\
\hline Até 1,60 & 92 & 25 & 1,0 & \\
\hline 1,61 a 1,65 & 63 & 15 & 0,75 & $0,37-1,75$ \\
\hline 1,66 e mais & 138 & 19 & 0,32 & $0,15-0,68$ \\
\hline \multicolumn{5}{|c|}{ Renda em salários mínimos } \\
\hline 5 a 10 & 111 & 15 & 1,0 & \\
\hline 11 a 20 & 111 & 26 & 2,00 & $0,93-4,23$ \\
\hline Mais de 20 & 58 & 16 & 2,87 & $1,23-6,67$ \\
\hline \multicolumn{5}{|c|}{$\begin{array}{l}\text { Número de pacientes atendidos } \\
\text { por } 4 \text { horas }\end{array}$} \\
\hline Até 4 & 134 & 24 & 1,0 & \\
\hline 5 a 6 & 128 & 26 & 1,15 & $0,58-2,26$ \\
\hline 7 ou mais & 31 & 11 & 3,28 & $1,29-8,35$ \\
\hline
\end{tabular}


tes com aqueles encontrados em outros estudos, incluindo fatores importantes em saúde ocupacional, como o ruído (compressor), aspectos da organização do trabalho como a produtividade, características individuais e psicossociais (Buckle, 1997).

Os estudos epidemiológicos recentes confirmam a relação dos movimentos de esforço, repetição e sobrecarga estática na origem de muitos problemas músculo-esqueléticos (Bernard, 1997; Buckle, 1997). Entre os mecanismos fisiopatológicos referidos na gênese desses problemas, englobam-se contrações contínuas e aumento de pressão intramuscular, interrupção do aporte sangüíneo e compressões de feixes nervosos, levando a sofrimento muscular crônico (Buckle, 1997). Essas condições parecem explicar tanto a associação com a exposição no trabalho, como outras atividades que envolvam esforço semelhante.

A maior prevalência de dor entre dentistas expostos ao barulho dos compressores pode ser compreendida dentro da perspectiva do ruído enquanto fonte de sobrecarga (Wickstrom \& Pentti, 1998), capaz de desencadear desequilíbrio fisiológico, gerando irritabilidade, estresse e tensões musculares mesmo em níveis inferiores (< 80 decibéis) aos causadores de lesões auditivas (Azevedo et al., 1993; Ferreira, 1997a, 1997b).

O tipo de visão utilizada pelos dentistas pode estar denotando a inter-relação entre instrumentos de trabalho, modo de realização da atividade e esforço físico. A visão indireta, isto é, utilizando-se o espelho durante os procedimentos, foi associada a menores freqüências de dor no pescoço, possivelmente porque o espelho facilita e ameniza os movimentos para exame da cavidade oral. A associação inversa entre queixa de dor no ombro e altura dos indivíduos poderia também ser explicada pelo menor esforço dos dentistas de maior estatura, requerido para o alcance e manuseio de instrumentos e do próprio paciente.

A relação inversa entre dor na coluna e prática de atividade manual pode estar simplesmente indicando que aqueles com dor na coluna tendem a realizar menos este tipo de atividade, possivelmente em virtude da própria dor. Todavia, dada a natureza seccional do estudo, não é possível esclarecer esta questão.

Fatores psicossociais ligados à organização do trabalho e fatores psicológicos individuais podem estar presentes e imersos entre condições especiais de exposição, nesse caso relacionadas às exigências do processo de trabalho dos dentistas (Cooper et al., 1987; Coster et al., 1987; Rundcrantz et al., 1990, 1991a, 1991b;
Murtomaa et al., 1990; Letho et al., 1991; Mandel, 1993; Turley et al., 1993; Doorn, 1995; Freeman et al., 1995a, 1995b; Derebery, 1998; Shelerud, 1998; Stutts \& Kasdan, 1998; Wickstrom \& Pentti, 1998). Esses fatores são apontados como indicadores de estresse, o que reforça a idéia da odontologia como uma profissão física e mentalmente estressante e sua associação com problemas músculo-esqueléticos (Murtomaa et al., 1990; Letho et al., 1991). Em nosso estudo, os indivíduos com facilidade de depressão, ansiedade e/ou preocupação excessiva apresentaram maior freqüência de dor na coluna e pescoço. Por outro lado, maior satisfação no trabalho esteve associada à menor freqüência de dor no pescoço. De acordo com alguns estudos (Bernard, 1997), há poucas evidências de uma associação importante entre as características psicológicas individuais e as altas taxas de distúrbios músculo-esqueléticos. Por outro lado, os fatores psicossociais parecem contribuir para os sintomas osteomusculares, independentemente dos perfis de personalidade (Pocekay et al., 1995; Schierhout \& Myers, 1996).

De qualquer modo, a relação entre alguns dos fatores psicossociais e psicológicos e as manifestações osteomusculares englobadas como DORT está permeada pelo velho dilema da incerteza temporal das variáveis analisadas (Kelsey, 1982; Wickstrom \& Pentti, 1998), não sendo possível dizer, com base nos estudos transversais, se os fatores analisados antecedem ou sucedem o início da dor. É possível, inclusive, que façam parte do próprio quadro clínico ou da sua evolução, questão que também não poderá ser esclarecida em estudos com esse desenho. Apesar da complexidade exigida para analisar as representações sobre satisfação no trabalho (Assunção et al., 1995), a associação entre maior satisfação e menor freqüência de dor no pescoço pode ser uma indicação de que os indivíduos mais satisfeitos estariam melhor adaptados ao trabalho, com menor grau de tensão muscular localizada. Ao mesmo tempo, é possível pensar que aqueles que sofrem de dores constantes são mais insatisfeitos com o trabalho, devido à própria dificuldade de adaptação por causa da dor.

Alguns estudos identificaram como preditores de dor a insatisfação com a condição de assalariamento e com a remuneração (Rundcrantz et al., 1990; Letho et al., 1991). Nossos resultados destoam desses achados, uma vez que a satisfação com a remuneração não apresentou relação com a queixa de dor e o nível salarial mais elevado foi associado à maior freqüência de dor no ombro. É possível pensar que 
uma renda maior pode ser indicativa de maiores jornadas de trabalho, o que no entanto não pôde ser averiguado pela dificuldade de mensurar a jornada dos profissionais fora do setor público. Por outro lado, um potencial fenômeno de confusão com o nível de produtividade dos dentistas foi descartada, já que ambas as variáveis (maior renda e maior produtividade) permaneceram associadas à maior freqüência de dor no ombro na análise multivariada.

Este estudo obteve uma taxa de participação bastante elevada (92,3\%), o que confere validade interna ao inquérito. Seria importante pesquisar a dor osteomuscular em dentistas que não trabalham no serviço público para verificar se as altas taxas de prevalência observadas entre os dentistas deste estudo refletem a magnitude do problema na categoria como um todo.

Os quadros clínicos de membros superiores em dentistas, como em outros trabalhadores, manifestam-se com ampla diversidade de apresentação (Moreira \& Carvalho, 1996; Bernard, 1997; Santos Filho \& Barreto, 1998a), como mostram nossos achados de intensidade e espectro de duração da dor, de clara tendência à cronificação, confirmados pela gama de diagnósticos distintos informados pelos dentistas que procuraram assistência médica. Ainda não existem métodos diagnósticos sensíveis ou específicos que permitam padronizar a definição de caso e orientar os estudos desses problemas. Intervalos de freqüência da dor e indicadores indiretos de gravidade, como as suas repercussões no cotidiano das pessoas, têm sido utilizados para caracterizar casos e não-casos (Westgaard \& Jansen, 1992; Pocekay et al., 1995; Baron et al., 1996; Punnett, 1996; Viikari-Juntura et al., 1996). Outros critérios seriam as tendências evolutivas (temporais) de redução, estabilidade ou agravamento e necessidade de medicação, além de atributos de funcionalidade e conseqüências sociais da dor, como limitações no trabalho e índice de absenteísmo e afastamentos. Todas essas questões foram importantes para a caracterização da queixa de dor osteomuscular entre os participantes deste estudo. Porém, optamos por não utilizar esses parâmetros para classificar casos e não-casos na análise de fatores associados, considerando não somente a complexidade dos elementos subjetivos embutidos na informação sobre intensidade e restrições impostas pela dor, mas também devido à insipiência de estudos de validação sobre os diversos critérios de classificação encontrados na literatura (Westgaard \& Jansen, 1992; Raspe \& Kohlmann, 1994; Stutts \& Kasdam, 1998). Optamos, portanto, por uma análise conservadora, visto que o efeito de uma possível inclusão de não-casos entre os casos tenderia a diluir uma associação aproximandoa da unidade.

No que diz respeito à localização física dos sintomas, é evidente a relação de contigüidade entre as regiões do segmento superior do corpo, estando essas regiões numa relação de prolongamento anátomo-funcional, dificultando, às vezes, a delimitação precisa do local e extensão da queixa. Apesar de a possibilidade dos diversos fatores de exposição no trabalho atuarem simultaneamente em mais de uma estrutura anatômica (Schierhout \& Myers, 1996), os estudos realizados têm indicado fatores etiológicos específicos para os diversos processos localizados (Kelsey, 1982). A clínica dos DORT também parece reforçar a importância da abordagem regional da dor, uma vez que quanto mais significativos os processos fisiopatológicos, mais bem localizados eles tendem a se apresentar (G. A. Ferreira, comunicação pessoal). Além disso, nossos achados de comorbidade entre as queixas regionais indicaram uma certa independência da dor em cada local, com percentuais de 9 a $29 \%$ de dor em duas regiões, ao contrário de achados de outros estudos de até $90 \%$ de dor combinada (Raspe \& Kohlmann, 1994). Isso reforçou nossa decisão de trabalhar a análise por região em separado.

A questão da temporalidade é uma limitação metodológica importante explicada pela natureza transversal deste e da grande maioria dos estudos realizados sobre as desordens osteomusculares de membros superiores (Bernard, 1997; Santos Filho \& Barreto, 1998a). No nosso estudo, a dor foi muito diversificada em duração e estaria muito provavelmente atravessada por mudanças de comportamento e hábitos, relatados como modificações na forma de trabalho, lazer e rotinas pessoais. Mudanças na exposição condicionadas pela presença do sintoma dificultam a identificação dos fatores precipitadores e daqueles associados ao prognóstico ou evolução dos quadros de dor. Os resultados da análise dos fatores preditores em cada região devem ser interpretados com cuidado, não apenas devido à possibilidade da dor crônica modificar a exposição dentro e fora do trabalho, mas também devido à metodologia de análise, com exploração repetida dos dados, o que eleva a chance de serem detectadas associações significativas quando elas não existem (Pocekay et al., 1995; Clayton \& Hills, 1996).

Uma outra fonte potencial de viés refere-se à confiabilidade e validade das informações de dor obtidas por questionário auto-aplicável (Westgaard \& Jansen, 1992; Raspe \& Kohlmann, 1994; Baron et al., 1996; Schierhout \& Myers, 
1996; Viikari-Juntura et al., 1996; Toomingas et al., 1997). Os problemas em torno das medidas subjetivas podem ser agrupados em duas grandes vertentes (Schierhout \& Myers, 1996): os relacionados aos padrões de apresentação do sintoma, ressaltando-se toda a atipia nas manifestações e testes diagnósticos de dor músculo-esquelética; e os fatores que influenciam a percepção e relato, lembrando que a experiência de dor encontra-se relacionada tanto a complexos mecanismos neuro-musculares quanto a fatores psicológicos individuais e psicossociais, como insatisfação no trabalho e estresses diversos, até o contexto social no qual ocorre o diagnóstico (Cole \& Hudak, 1996; Schierhout \& Myers, 1996). Contudo, considerando que os testes clínico-laboratoriais são imprecisos, principalmente para casos discretos e iniciais, inclusive para aqueles diagnósticos fechados, como síndrome do túnel do carpo, os estudos indicam a dor auto-referida como uma medida importante e válida para inquéritos (Westgaard \& Jansen, 1992; Schierhout \& Myers, 1996). Para minimizar o viés de informação, recomenda-se que a abordagem seja mais direcionada quanto à delimitação regional do sintoma (Raspe \& Kohlmann, 1994; Schierhout \& Myers, 1996). A qualidade da informação parece aumentar quando as localizações da dor são deixadas em aberto (Schierhout \& Myers, 1996), como fizemos neste estudo. Parece também haver uma correlação entre relatos de quadros mais graves e maiores prevalências de achados ao exame físico, crescendo também a procura de assistência médica de acordo com essa gravidade (Westgaard \& Jansen, 1992; Baron et al., 1996), o que também reforça a adequação do instrumento usado por nós.

A consistência dos resultados deste trabalho com o de outros inquéritos realizados na categoria apontam a necessidade de discutir a insuficiência das intervenções já realizadas no mobiliário e método de trabalho dos dentistas, como modificações nos equipamentos (Rundcrantz et al., 1990; Mandel, 1993; Doorn, 1995). Por exemplo, entre as desvantagens do trabalho sentado, sobretudo por longos períodos, pode-se citar o aumento de pressão nos discos intervertebrais, fator de risco conhecido para dor lombar (Kelsey, 1982; Mandel, 1993). Portanto, se o trabalho sentado é, em princípio, mais confortável, pode-se tornar mais danoso se é realizado sem cuidados com a postura (Ferreira, 1997a, 1997b) ou sem controle de outros fatores da organização do trabalho, como jornadas e ritmo. De modo semelhante, pode ser mais significativa a forma como os dentistas manuseiam (se ajustam ou não) os equipamen- tos de trabalho (cadeira e equipos), independentemente da sofisticação tecnológica que eles apresentam. Por outro lado, há experiências mostrando que algumas intervenções não reduziram a incidência de problemas, mas afetaram positivamente a evolução dos casos, resultando em impacto quanto à sua gravidade (Baron et al., 1996). Tais questões devem orientar a reflexão em torno do trabalho em odontologia, subsidiando avaliações de riscos e propostas efetivas de mudanças no trabalho.

\section{Conclusão}

Este estudo apresenta um inquérito epidemiológico da queixa de dor osteomuscular no segmento superior em cirurgiões-dentistas no nosso meio, trazendo uma exploração inicial dos fatores associados a essas queixas. Na presente investigação, contou-se com uma elevada taxa de participação dos trabalhadores elegíveis (92,3\%), salientando-se também o pequeno percentual de respostas em branco e a consistência interna das mesmas, situação que não é usual em inquéritos com questionário auto-aplicável, principalmente em países como o Brasil (Raspe \& Kohlmann, 1994; Bernard, 1997). Os resultados obtidos indicam a necessidade de estudos futuros que aprofundem a abordagem da queixa por região, incorporando entrevistas, exames clínicos e complementares e realizando uma exploração longitudinal da história ocupacional e de outras exposições relevantes. Acreditamos que os resultados deste estudo são particularmente relevantes para os próprios cirurgiõesdentistas, incentivando e orientando o debate sobre os DORT na categoria e a discussão de possíveis medidas de prevenção. 


\section{Agradecimentos}

A colaboração dos doutores Gilda A. Ferreira, Francisco P. A. Lima, Renato C. Ferreira e Izabela Pordeus foi fundamental para a realização do estudo, especialmente para a elaboração do questionário utilizado. O Dr. Joaquim S. Martins Júnior colaborou imensamente durante o processamento dos dados. Esta investigação só foi possível pelo apoio da Coordenação de Odontologia da Secretaria de Saúde de Belo Horizonte, bem como de todas as Referências de Odontologia, Diretores, Chefias e demais trabalhadores dos Distritos Sanitários. Agradecimento especial aos dentistas envolvidos nas estratégias de pesquisa de campo (Silene, Beatriz e Eugênio) e a todos que participaram do estudo.

\section{Referências}

AKESSON, I.; LUNDBORG, G.; HORSTMANN, V. \& SKERFVING, S., 1995. Neuropathy in female dental personnel exposed to high frequency vibrations. Occupational and Environmental Medicine, 52:116-123.

ASSUNÇÃO, A. A.; NUNES, C. M. P.; LIMA, F. P. A.; ALVES, G. B. O.; ARAÚJO, J. N. G.; MONTEIRO, L. S. S.; VECHIA, M. L. D.; LIMA, M. E. A; OLIVEIRA, P. L. \& SOUZA, R. J., 1995. A Produção das Lesões por Esforços Repetitivos no Restaurante Universitário Setorial II: Análise Ergonômica e Psicossocial. Relatório Final, Belo Horizonte: Núcleo de Estudos em Saúde e Trabalho Humano, Universidade Federal de Minas Gerais.

AZEVEDO, A. P.; MARATA, T. C.; OKAMATO, V. A. \& SANTOS, U. P., 1993. Ruído: Um problema de saúde pública (outros agentes físicos). In: Isto é Trabalho de Gente? Vida, Doença e Trabalho no Brasil (J. T. Buschinelli, L. E. Rocha \& R. M. Rigotto, org.), pp. 403-435, São Paulo: Editora Vozes.

BAMMER, G., 1996. Work-related neck and upper limb disorders: Social, organisational, biomechanical and medical aspects. In: I Seminário Internacional Fiemg de Saúde Ocupacional/Lesões por Esforços Repetitivos/LER, Belo Horizonte.

BARON, S.; HALES, T. \& HURREL, J., 1996. Evaluation of symptom surveys for occupational musculoskeletal disorders. American Journal of Industrial Medicine, 29:609-617.

BAUER, M. E., 1985. Carpal Tunnel Syndrome: An occupational risk to the dental hygienist. Dental Hygiene, 59:218-221.

BERNARD, B. P., 1997. Musculoskeletal Disorders (MSDs) and Workplace Factors: A Critical Review of Epidemiologic Evidence for Work-Related Musculoskeletal Disorders of the Neck, Upper Extremity, and Low Back. 24 October $2000<$ http: / /www. cdc.gov/niosh/ergoscil.html>.

BUCKLE, P., 1997. Work factors and upper limb disorders. BMJ, 315:1360-1363.
CDC (Centers for Disease Control and Prevention)/ WHO (World Health Organization), 1996. Epi Info 6, Version 6.04. A Word Processing, Database, and Statistics Program for Public Health. Atlanta: CDC/ Geneva: WHO.

CHECKOWAY, H.; PEARCE, N. E. \& CRAWFORDBROWNE, D. J., 1989. Research Methods in Occupational Epidemiology. New York: Oxford University Press.

CHERNIACK, M. G., 1996. Epidemiology of occupational disorders of the upper extremity. Occupational Medicine: State of the Art Reviews, 11:513530.

CLAYTON, D. \& HILLS, M., 1996. Statistical Models in Epidemiology. Oxford/New York/Tokyo: Oxford University Press.

COLE, D. C. \& HUDAK, P. L., 1996. Prognosis of nonspecific work-related musculoskeletal disorders of the neck and upper extremity. American Journal of Industrial Medicine, 29:657-668.

COOPER, C. L.; WATTS, J. \& KELLY, M., 1987. Job satisfaction, mental health, and job stressors among general dental practitioners in the United Kingdom. British Dental Journal, 162:77-81.

COSTER, E. A.; CARSTENS, I. L. \& HARRIS, A. M. P., 1987. Patterns of stress among dentists. Journal of the Dental Association of South Africa, 42:389394.

DEREBERY, V. J., 1998. Determining the cause of upper extremity complaints in the workplace. Occupational Medicine: State of the Art Reviews, 13: 569-582.

DOORN, J. W. C., 1995. Low back disability among self-employed dentists, veterinarians, physicians and physical therapists in the Netherlands. Acta Orthopaedica Scandinavica, 66:1-64.

EKENVALL, L.; NILSSON, B. Y. \& FALCONER, C., 1990. Sensory perception in the hands of dentists. Scandinavian Journal of Environmental Health, 16:334-339. 
FERREIRA, R. C., 1997a. As Posições de Trabalho para Profissionais de Saúde Bucal. Belo Horizonte: Ministério da Saúde.

FERREIRA, R. C., 1997b. Agentes de Carga no Trabalho em Odontologia e Principais Formas de Prevenção. Belo Horizonte: Ministério da Saúde.

FREEMAN, R.; MAIN, J. R. R. \& BURKE, F. J. T., 1995a. Occupational stress and dentistry: Theory and practice. Part I - Recognition. British Dental Journal, 178:214-217.

FREEMAN, R.; MAIN, J. R. R. \& BURKE, F. J. T., 1995b. Occupational stress and dentistry: Theory and practice. Part II - Assessment and control. British Dental Journal, 178:218-222.

INSS (Instituto Nacional de Securidade Social), 1998. Distúrbios Osteomusculares Relacionados ao Trabalho (DORT). Brasília: INSS.

KELSEY, J. L., 1982. Epidemiology of Musculoskeletal Disorders. New York: Oxford University Press.

LETHO, T. U.; HELENIUS, H. Y. M. \& ALARANTA, H. T., 1991. Musculoskeletal symptoms of dentists assessed by a multidisciplinary approach. Community Dentistry and Oral Epidemiology, 19:38-44.

LETHO, T. U.; RONNEMAA, T.; AALTO, T. \& HELENIUS, H. Y. M., 1990. Roentgenological arthrosis of the hand in dentists with reference to manual function. Community Dentistry and Oral Epidemiology, 18:37-41.

LOPES, A. \& NETO, R. V., 1994. A Síndrome do túnel carpal: Um risco profissional para o cirurgiãodentista. Revista da Associação Paulista de Cirurgiões Dentistas, 48:1545-1552.

MANDEL, I. D., 1993. Occupational risks in dentistry: Comforts and concerns. Journal of the American Dental Association, 124:41-49.

MEDEIROS, U. V. \& RIUL, L. F., 1994. Riscos ocupacionais do cirurgião-dentista e sua prevenção. Revista Paulista de Odontologia, 6:34-43.

MILERAD, E. \& EKENVALL, L., 1990. Symptoms of the neck and upper extremities in dentists. Scandinavian Journal of Work, Environment and Health, 16:129-134

MOREIRA, C. \& CARVALHO, M. A. P., 1996. Noções Práticas de Reumatologia. Belo Horizonte: Editora Health.

MURTOMAA, H.; HAAVIO-MANNILA, E. \& KANDOLIN, I., 1990. Burnout and its causes in Finish dentists. Community Dentistry and Oral Epidemiology, 18:208-212.

NOGUEIRA, D. P., 1983. Riscos ocupacionais de dentistas e sua prevenção. Revista Brasileira de Saúde Ocupacional, 41:16-24.

POCEKAY, D.; McCURDY, S. A.; SAMUELS, S. J.; HAMMOND, S. K. \& SCHENCKER, M. B., 1995. A crossseccional study of musculoskeletal symptoms and risk factors in semiconductor workers. American Journal of Industrial Medicine, 28:861-871.

PUNNETT, L., 1996. Adjusting for the healthy worker selection effect in cross-seccional studies. International Journal of Epidemiology, 25:1068-1076.

RASPE, H. \& KOHLMANN, T., 1994. Disorders characterised by pain: A methodological review of population surveys. Journal of Epidemiology and Community Health, 48:531-537.

RUNDCRANTZ, B. L.; JOHNSSON, B. \& MORITZ, U., 1990. Cervical pain and discomfort among den- tists: Epidemiological, clinical and therapeutic aspects. Part 1: A survey of pain and discomfort. Swedish Dental Journal, 14:81-89.

RUNDCRANTZ, B. L.; JOHNSSON, B. \& MORITZ, U., 1991a. Pain and discomfort in the musculoskeletal system among dentists. A prospective study. Swedish Dental Journal, 15:219-228.

RUNDCRANTZ, B. L.; JOHNSSON, B. \& MORITZ, U., 1991b. Occupational cervico-brachial disorders among dentists. Analysis of ergonomics and locomotor functions. Swedish Dental Journal, 15: 105-115.

SANTOS FILHO, S. B. \& BARRETO, S. M., 1998a. Algumas considerações metodológicas sobre os estudos epidemiológicos das Lesões por Esforços Repetitivos (LER). Cadernos de Saúde Pública, 14:555-563.

SANTOS FILHO, S. B. \& BARRETO, S. M., 1998b. Sintomas Músculo-esqueléticos em Cirurgiões-dentistas de Minas Gerais: Epidemiologia das Lesões por Esforços Repetitivos. Belo Horizonte: Sindicato dos Odontologistas de Minas Gerais.

SCHIERHOUT, G. H. \& MYERS, J. E., 1996. Is self-reported pain an appropriate outcome measure in ergonomic-epidemiologic studies of work-related musculoskeletal disorders? American Journal of Industrial Medicine, 30:93-98.

SHELERUD, R., 1998. Epidemiology of occupational low back pain. Occupational Medicine: State of the Art Reviews, 13:01-22.

STATA CORPORATION, 1997. Stata Statistical Software. Release 5.0. College Station: Stata Corporation.

STUTTS, J. \& KASDAN, M., 1998. Psychosocial aspects of hand injuries and diseases. Occupational Medicine: State of the Art Reviews, 13:513-519.

TOOMINGAS, A.; ALFREDSSON, L. \& KILBOM, A., 1997. Possible bias from rating behavior when subjects rate both exposure and outcome. Scandinavian Journal of Work, Environment and Health, 23:370-377.

TRAVASSOS, D. V., 1995. A Equipe Odontológica e as Doenças Profissionais. Monografia, Belo Horizonte: Faculdade de Odontologia, Universidade Federal de Minas Gerais.

TURLEY, M.; KINIRONS, M. \& FREEMAN, R., 1993. Occupational stress factors in hospital dentists. British Dental Journal, 175:285-288.

VIIKARI-JUNTURA, E.; RAUAS, S.; MARTIKAINEN, R.; KUOSMA, E.; RIIHIMAKI, H.; TAKALA, E.-P. \& SAARENMAA, K., 1996. Validity of self-reported physical work load in epidemiological studies on musculoskeletal disorders. Scandinavian Journal of Work, Environment and Health, 22:251-259.

WESTGAARD, R. H. \& JANSEN, T., 1992. Individual and work related factors associated with symptoms of musculoskeletal complaints. I - A quantitative registration system. British Journal of Industrial Medicine, 49:147-153.

WICKSTROM, G. J. \& PENTTI, J., 1998. Occupational factors affecting sick leave attributed to low-back pain. Scandinavian Journal of Work, Environment and Health, 24:145-152. 